\title{
GnRH Antagonist Protocol: Is It Effective for Expected Poor Ovarian Responders with Tubal Factor Undergoing IVF?
}

\author{
Weijie Xing1, Haiyan Lin², Qingxue Zhang2* \\ ${ }^{1}$ Center for Reproductive Medicine, The Third Affiliated Hospital of Sun Yat-sen University, Guangzhou, China \\ ${ }^{2}$ Center for Reproductive Medicine, Sun Yat-sen Memorial Hospital of Sun Yat-sen University, Guangzhou, China \\ Email: *zhangqingxue666@aliyun.com
}

How to cite this paper: Xing, W.J., Lin, H.Y. and Zhang, Q.X. (2017) GnRH Antagonist Protocol: Is It Effective for Expected Poor Ovarian Responders with Tubal Factor Undergoing IVF? Advances in Reproductive Sciences, 5, 57-63.

https://doi.org/10.4236/arsci.2017.54007

Received: September 14, 2017

Accepted: October 22, 2017

Published: October 25, 2017

Copyright $\odot 2017$ by authors and Scientific Research Publishing Inc. This work is licensed under the Creative Commons Attribution International License (CC BY 4.0).

http://creativecommons.org/licenses/by/4.0/

\begin{abstract}
Background: This study aimed to determine if the gonadotropin releasing hormone (GnRH) antagonist protocol is optimal for expected poor ovarian responders with tubal factor undergoing in vitro fertilization-embryo transfer (IVF-ET). Methods: A total of 341 IVF-ET cycles were retrospectively identified. The following inclusion criteria were applied: age $\geq 40$ years and patients with tubal factors. The cycles were divided into two groups: a GnRH antagonist group (157 cycles) and a GnRH agonist group (184 cycles). Results: The duration of stimulation and the total doses of gonadotropin in the GnRH agonist group were significantly more than those in the GnRH antagonist group ( $F$ $<0.05)$. There were significant differences in LH and $P$ values on the hCG measurement days between the two groups $(0.91 \pm 1.17$ vs. $4.82 \pm 4.69 \mathrm{U} / \mathrm{L}$ and $0.69 \pm 0.42$ vs. $1.03 \pm 0.50 \mathrm{ng} / \mathrm{mL}, P<0.05)$. The implantation rate of the GnRH antagonist group was $12.24 \%$, which was slightly higher than that of the GnRH agonist group $(10.10 \%, P=0.437)$. The clinical pregnancy rate of the two groups showed no statistical differences $(23.36 \%$ vs. $23.03 \%, P=1.000)$. Conclusion: For expected poor ovarian responders, the GnRH antagonist protocol was, to some extent, superior to the GnRH agonist protocol in terms of the implantation and clinical pregnancy rates.
\end{abstract}

\section{Keywords}

Poor Ovarian Responders, GnRH Antagonist Protocol, GnRH Agonist Protocol

\section{Background}

During controlled ovarian stimulation (COS) for assisted reproductive technologies (ARTs), poor ovarian response (POR) to gonadotropin (Gn) stimulation is 
not uncommon [1]. The incidence of POR has been reported to vary between $9 \%$ and 24\% [2]. In 2011, the Bologna criteria for POR were raised and have been recently recognized. According to the Bologna criteria, POR should be diagnosed if the patients have two out of the following three features: 1$)$ advanced age ( $\geq 40$ years) or any other risk factor for POR; 2) a history of POR (three or fewer oocytes retrieved with ovulation induction); or 3) lower ovarian reserve [antral follicular count (AFC) less than 5 - 7 follicles or Anti-Mullerian hormone (AMH) level less than $0.5 \mathrm{ng} / \mathrm{mL}$ to $1.1 \mathrm{ng} / \mathrm{mL}$ ] [3].

Gonadotropin-releasing hormone $(\mathrm{GnRH})$ agonists have been successfully used for the last 20 years [4]. Hypophyseal desensitization can be induced by the administration of GnRH agonists, which can prevent premature luteinization and then significantly reduce the cycle cancellation rate via the nearly complete inhibition of spontaneous LH surges $(<2 \%)$ [5]. Because the long protocol of $\mathrm{GnRH}$ agonists has been found to be superior to other protocols, it is most frequently used throughout the world. However, for POR, extremely higher doses of Gn and longer durations of stimulation are often required due to over-suppression [6]. The cycle cancellation rate is also fairly high for POR with the GnRH agonist long protocol.

For POR, selecting an optimal protocol for IVF/ICSI cycles is still a frustrating challenge. Numerous strategies have been suggested to improve the outcome of POR, such as the GnRH antagonist (GnRHant) protocol, minimal ovarian stimulation protocol, Shanghai protocol, and so on [1] [7] [8] [9]. The GnRHant protocol, which was first introduced in the late 1990s, may be an effective protocol for POR because of its distinctive pharmacologic properties. Currently, GnRH antagonists are mainly used in many clinics for patients with PCOS undergoing IVF. However, even though it can significantly lower the incidence of OHSS, the lower implantation and pregnancy rate limit the application of the GnRHant protocol.

There is still no consensus on which protocol is the most effective and safe one for POR. The aim of this study was to compare the effectiveness of the GnRHant protocol and GnRH agonist long protocol in expected POR with tubal factor during IVF cycles.

\section{Method}

A total of 341 IVF-ET cycles from January 2011 to December 2014 were retrospectively identified in the Center for Reproductive Medicine of Sun Yat-sen Memorial Hospital of Sun Yat-sen University. The following inclusion criteria were applied: 1) age $\geq 40$ years; and 2) patients with tubal factors. The cycles were divided into two groups accordingly: a GnRHant group (157 cycles) and a GnRHa group (184 cycles). This retrospective study was approved by the Sun Yat-sen Memorial Hospital Medicine Ethics Committee. Patient records and information were anonymized and de-identified prior to analysis.

In the GnRHant group, the protocol was the same as previously described [10]. 
Ovarian stimulation commenced with 150 - $450 \mathrm{IU}$ rFSH from day 2 to 3 of the menstrual cycle. When the serum $\mathrm{E}_{2}$ level was higher than $300 \mathrm{pg} / \mathrm{mL}$, or a lead follicle reached a mean diameter of $14 \mathrm{~mm}$, a daily dose of $0.25 \mathrm{mg}$ GnRHant (Cetrotide, Serono, Switzerland) was initiated and continued until the day of hCG administration. When at least two follicles reached a mean diameter of 18 $\mathrm{mm}$, recombinant hCG (Serono, Switzerland) was given to trigger follicle maturation. Oocyte retrieval was performed transvaginally 34 - 36 hours later.

In the GnRHa group, down-regulation treatment with 1.0 - $1.3 \mathrm{mg}$ of Triptorelin (3.75 mg Gonapeptyl; Ferring) was started from day 20 of the preceding menstrual cycle. In accordance with body mass index and patient age, recombinant FSH (Gonal-F, Serono, Switzerland) and/or hMG (Li Zhu, China) were used at dosages ranging between $150 \mathrm{IU} /$ day and $450 \mathrm{IU} /$ day. The timing of the hCG trigger was similar to the $\mathrm{GnRH}$ agonist protocol. Oocyte retrieval was carried out 34 - 36 hours after triggering by transvaginal ultrasound-guided puncture.

The embryo transfers (ET) were performed three to five days later under ultrasound guidance. The thickness of the endometrium less than $6 \mathrm{~mm}$ was considered too thin. The cycles were cancelled when the endometrium was too thin or there were no dominant follicles (diameter less than $14 \mathrm{~mm}$ ). Pregnancy was diagnosed by an increase in the concentration of serum $\beta$-hCG, which was tested 14 days after ET. Clinical pregnancy was defined as the presence of a gestational sac on vaginal ultrasound examination. Miscarriage was defined as a pregnancy loss before 28 weeks [11].

\section{Statistical Analysis}

SPSS statistical software package (version 11.0) was used for statistical analysis. Values are expressed as the mean \pm SD. The unpaired Student's $t$-test was used to compare means from two groups. The $\chi^{2}$-test was used to compare categorical variables. $P<0.05$ was considered statistically significant.

\section{Results}

A total of 341 IVF-ET cycles were retrospectively studied. The patient demographic variables are compared in Table 1. The two groups were similar in terms of age, basal FSH, body mass index, proportion of primary infertility, and duration of infertility. The duration of stimulation and the total doses of $\mathrm{Gn}$ in the GnRHa group were $11.51 \pm 1.46$ days and $3317.34 \pm 775.29$ IU, respectively, which were significantly more than those in the GnRHant group (9.70 \pm 2.23 days and $2306.88 \pm 757.22 \mathrm{IU}, P<0.05)$. There were significant differences in LH and P levels on the hCG day between the two groups $(0.91 \pm 1.17$ vs. $4.82 \pm$ $4.69 \mathrm{U} / \mathrm{L}$ and $0.69 \pm 0.42$ vs. $1.03 \pm 0.50 \mathrm{ng} / \mathrm{mL}, P<0.05)$. However, the $\mathrm{E}_{2}$ levels on the hCG day were similar between the two groups $(2095.28 \pm 1213.17$ vs. $1953.47 \pm 1248.00 \mathrm{pg} / \mathrm{mL}, P=0.361)$.

As shown in Table 2, the number of oocytes retrieved in the GnRHant group was $6.37 \pm 4.26$, which was significantly less than the number retrieved in the 
Table 1. Epidemiologic and stimulation characteristics.

\begin{tabular}{cccc}
\hline Variable & GnRHa $(\mathrm{N}=184)$ & GnRHant $(\mathrm{N}=157)$ & $P$ value \\
\hline Age (years) & $41.52 \pm 1.56$ & $41.48 \pm 1.66$ & 0.781 \\
Basal FSH (IU/L) & $10.16 \pm 4.87$ & $11.17 \pm 6.34$ & 0.198 \\
Body mass index (kg/m $\left.{ }^{2}\right)$ & $22.40 \pm 2.48$ & $22.38 \pm 3.23$ & 0.953 \\
Proportion of primary infertility (\%) & $34.87 \%$ & $31.78 \%$ & 0.645 \\
Duration of infertility (years) & $6.78 \pm 4.77$ & $6.21 \pm 5.12$ & 0.353 \\
Duration of stimulation (days) & $11.51 \pm 1.46$ & $9.70 \pm 2.23$ & $<0.05$ \\
Total dose of Gn administered (IU) & $3317.34 \pm 775.29$ & $2306.88 \pm 757.22$ & $<0.05$ \\
E level on hCG trigger day (pg/mL) & $2095.28 \pm 1213.17$ & $1953.47 \pm 1248.00$ & 0.361 \\
LH level on hCG trigger day (mIU/mL) & $0.91 \pm 1.17$ & $4.82 \pm 4.69$ & $<0.05$ \\
P level on hCG trigger day (ng/mL) & $0.69 \pm 0.42$ & $1.03 \pm 0.50$ & $<0.05$ \\
Endometrial thickness on hCG trigger day (mm) & $10.89 \pm 2.47$ & $10.39 \pm 2.35$ & 0.099 \\
\hline
\end{tabular}

Note: $\mathrm{NS}=$ not statistically significant. Values presented as mean \pm SD unless otherwise specified.

Table 2. IVF outcomes.

\begin{tabular}{cccc}
\hline Variable & $\begin{array}{c}\text { GnRHa } \\
(\mathrm{N}=184)\end{array}$ & $\begin{array}{c}\text { GnRHant } \\
(\mathrm{N}=157)\end{array}$ & P value \\
\hline No. of oocytes retrieved & $10.38 \pm 5.71$ & $6.37 \pm 4.26$ & $<0.05$ \\
& $32 / 184$ & $50 / 157$ & \\
No. of cancelled fresh transferred cycle (n, \%) & $(17.39 \%)$ & $(31.85 \%)$ & $<0.05$ \\
& $11 / 32$ & $12 / 50$ & 0.325 \\
No. of cancelled fresh transferred cycle because & $(34.38 \%)$ & $(24 \%)$ & \\
of no dominant follicles (n, \%) & $10.10 \%$ & $12.24 \%$ & 0.437 \\
Implantation rate (\%) & $35 / 152$ & $25 / 107$ & 1.000 \\
& $(23.03 \%)$ & $(23.36 \%)$ & \\
Clinical pregnancy/fresh transferred cycle (n, \%) & & & \\
& $13 / 35$ & $9 / 25$ & 1.000 \\
Miscarriage/fresh transferred cycle (n, \%) & $(37.14 \%)$ & $(36.00 \%)$ & \\
& & & \\
& &
\end{tabular}

Note: Values presented as mean \pm SD unless otherwise specified.

GnRHa group $(10.38 \pm 5.71, P<0.05)$. However, the numbers of high quality embryos and transferred embryos were similar in two groups $(2.21 \pm 1.65$ vs. $2.17 \pm 1.54,2.09 \pm 0.65$ vs. $2.03 \pm 0.33)$. In the GnRHa group, the rate of cancelled freshly transferred cycles was $17.39 \%$, which was significantly lower than that in the GnRHant group $(31.85 \%, P<0.05)$. The rate of cancelled freshly transferred cycles because of no dominant follicles in the GnRHa group was slightly higher than that in the GnRHant group (34.38\% vs. $24 \%, P=0.325)$.

When the $\chi^{2}$-test was performed, we obtained the following results: 1$)$ The implantation rate of the GnRHant group was $12.24 \%$, which was slightly higher than that of the GnRHa group $(10.10 \%, P=0.437) ; 2)$ there was no difference of the clinical pregnancy rate between two groups $(23.36 \%$ vs. $23.03 \%, P=1.000)$; 
and 3) there was no significant difference in the miscarriage rate between the two groups (36.00\% vs. $37.14 \%, P=1.000)$.

\section{Discussion}

Many studies demonstrated that, in poor ovarian responders with declining ovarian reserve, the expected pregnancy rate was dramatically lower than that of younger women [12]. The frequency of poor ovarian responder status has been estimated to be $10 \%$ in the general population and markedly higher in patients over 40 years of age [13]. For these patients, selection of an effective protocol remains a frustrating challenge in IVF cycles. The GnRH agonist protocol has been widely used in IVF centers. However, there are several disadvantages for the application of the GnRH-agonist long protocol in low responders, including increased $\mathrm{Gn}$ doses, the longer duration of Gn administration [14], the higher cycle cancellation rate, and the increased treatment costs [15]. Numerous strategies have been attempted to improve the outcome of POR, including: 1) increasing the doses of $\mathrm{Gn}[16]$; 2) application of other protocols [17]; 3) addition of growth hormone $(\mathrm{GH})$, and 4) addition of growth hormone-releasing factor (GHRF) [18].

However, most of these interventions have achieved extremely limited advantages; consequently, the optimal protocol for POR is still unknown [6]. The introduction of $\mathrm{GnRH}$ antagonists presented hope for POR. GnRH antagonists can induce pituitary suppression within a few hours without a "flare-up" effect, and the suppression can be released immediately after their discontinuation [19]. In the present study, we investigated the effectiveness of the GnRH antagonist protocol in expected poor ovarian responders. We found that the duration of stimulation and the total doses of $\mathrm{Gn}$ in the GnRHa group were $11.51 \pm 1.46$ days and $3317.34 \pm 775.29 \mathrm{IU}$, which were significantly more than those in the GnRHant group $(9.70 \pm 2.23$ days and $2306.88 \pm 757.22 \mathrm{IU}, P<0.05)$. These were in keeping with the results of previous studies. Several studies demonstrated a lower implantation rate and pregnancy rate in the $\mathrm{GnRH}$-ant protocol, which limited its wide application [20]. However, our study showed that, for expected POR (age $\geq 40$ years), the implantation rate and clinical pregnancy rate of the GnRHant group were slightly higher than those in the GnRHa group $(12.24 \%$ vs. $10.10 \%, P$ $=0.437 ; 23.36 \%$ vs. $23.03 \%, P=1.000)$. In our previous study, we demonstrated that, for potentially high responders, the GnRHa protocol was superior to the GnRHant protocol in terms of normal fertilization rate, implantation rates, and clinical pregnancy rates [10]. These findings showed that the GnRHant protocol may be an optimal protocol for expected poor ovarian responders, but not for potentially high responders.

There were some limitations because it was a retrospective study. The small sample size of the study should be noted. Our results should be confirmed by further, adequately sized studies, or meta-analyzed along with similar studies. 


\section{Conclusion}

In conclusion, our study demonstrated that for expected poor ovarian responders, the GnRH antagonist protocol was, to some extent, superior to the GnRH agonist protocol in terms of the duration of stimulation, the total doses of $\mathrm{Gn}$, the implantation rates and clinical pregnancy rates.

\section{Competing Interests}

The authors declare that they have no competing interests.

\section{References}

[1] Kucuk, T., Kozinoglu, H. and Kaba, A. (2008) Growth Hormone Co-Treatment within a GnRH Agonist Long Protocol in Patients with Poor Ovarian Response: A Prospective, Randomized, Clinical Trial. Journal of Assisted Reproduction and Genetics, 25, 123-127. https://doi.org/10.1007/s10815-008-9212-7

[2] Surrey, E.S. and Schoolcraft, W.B. (2000) Evaluating Strategies for Improving Ovarian Response of the Poor Responder Undergoing Assisted Reproductive Techniques. Fertility and Sterility, 73, 667-676. https://doi.org/10.1016/S0015-0282(99)00630-5

[3] Ferraretti, A.P., La Marca, A., Fauser, B.C., Tarlatzis, B., Nargund, G. and Gianaroli, L. (2011) ESHRE Consensus on the Definition of 'Poor Response' to Ovarian Stimulation for In Vitro Fertilization: The Bologna Criteria. Human Reproduction (Oxford, England), 26, 1616-1624. https://doi.org/10.1093/humrep/der092

[4] Hughes, E.G., Fedorkow, D.M., Daya, S., Sagle, M.A., Van de Koppel, P. and Collins, J.A. (1992) The Routine Use of Gonadotropin-Releasing Hormone Agonists Prior to In Vitro Fertilization and Gamete Intrafallopian Transfer: A Meta-Analysis of Randomized Controlled Trials. Fertility and Sterility, 58, 888-896. https://doi.org/10.1016/S0015-0282(16)55430-2

[5] Maldonado, L.G., Franco Jr., J.G., Setti, A.S., Iaconelli Jr., A. and Borges Jr., E. (2013) Cost-Effectiveness Comparison between Pituitary Down-Regulation with a Gonadotropin-Releasing Hormone Agonist Short Regimen on Alternate Days and an Antagonist Protocol for Assisted Fertilization Treatments. Fertility and Sterility, 99, 1615-1622. https://doi.org/10.1016/j.fertnstert.2013.01.095

[6] Cheung, L.P., Lam, P.M., Lok, I.H., Chiu, T.T., Yeung, S.Y., Tjer, C.C., et al. (2005) GnRH Antagonist versus Long GnRH Agonist Protocol in Poor Responders Undergoing IVF: A Randomized Controlled Trial. Human Reproduction (Oxford, England), 20, 616-621. https://doi.org/10.1093/humrep/deh668

[7] Malmusi, S., La Marca, A., Giulini, S., Xella, S., Tagliasacchi, D., Marsella, T., et al. (2005) Comparison of a Gonadotropin-Releasing Hormone (GnRH) Antagonist and GnRH Agonist Flare-Up Regimen in Poor Responders Undergoing Ovarian Stimulation. Fertility and Sterility, 84, 402-406. https://doi.org/10.1016/j.fertnstert.2005.01.139

[8] Schmidt, D.W., Bremner, T., Orris, J.J., Maier, D.B., Benadiva, C.A. and Nulsen, J.C. (2005) A Randomized Prospective Study of Microdose Leuprolide versus Ganirelix in In Vitro Fertilization Cycles for Poor Responders. Fertility and Sterility, 83, 1568-1571. https://doi.org/10.1016/j.fertnstert.2004.10.053

[9] Bosdou, J.K., Venetis, C.A., Kolibianakis, E.M., Toulis, K.A., Goulis, D.G., Zepiridis, L., et al. (2012) The Use of Androgens or Androgen-Modulating Agents in Poor Responders Undergoing In Vitro Fertilization: A Systematic Review and Meta-Analysis. 
Human Reproduction Update, 18, 127-145. https://doi.org/10.1093/humupd/dmr051

[10] Xing, W., Lin, H., Li, Y., Yang, D., Wang, W. and Zhang, Q. (2015) Is the GnRH Antagonist Protocol Effective at Preventing OHSS for Potentially High Responders Undergoing IVF/ICSI? PloS ONE, 10, e0140286.

https://doi.org/10.1371/journal.pone.0140286

[11] Ou, J., Xing, W., Li, Y., Xu, Y. and Zhou, C. (2015) Short versus Long Gonadotropin-Releasing Hormone Analogue Suppression Protocols in IVF/ICSI Cycles in Patients of Various Age Ranges. PLoS ONE, 10, e0133887.

https://doi.org/10.1371/journal.pone.0133887

[12] Assisted Reproductive Technology in the United States (2004) 2000 Results Generated from the American Society for Reproductive Medicine/Society for Assisted Reproductive Technology Registry. Fertility and Sterility, 81, 1207-1220.

[13] Sbracia, M., Farina, A., Poverini, R., Morgia, F., Schimberni, M. and Aragona, C. (2005) Short versus Long Gonadotropin-Releasing Hormone Analogue Suppression Protocols for Superovulation in Patients $>$ or $=40$ Years Old Undergoing Intracytoplasmic Sperm Injection. Fertility and Sterility, 84, 644-648.

[14] Hugues, J.N. and Cedrin Durnerin, I.C. (1998) Revisiting Gonadotrophin-Releasing Hormone Agonist Protocols and Management of Poor Ovarian Responses to Gonadotrophins. Human Reproduction Update, 4, 83-101.

https://doi.org/10.1093/humupd/4.1.83

[15] Ben-Rafael, Z., Lipitz, S., Bider, D. and Mashiach, S. (1991) Ovarian Hyporesponsiveness in Combined Gonadotropin-Releasing Hormone Agonist and Menotropin Therapy Is Associated with Low Serum Follicle-Stimulating Hormone Levels. Fertility and Sterility, 55, 272-275.

[16] Land, J.A., Yarmolinskaya, M.I., Dumoulin, J.C. and Evers, J.L. (1996) High-Dose Human Menopausal Gonadotropin Stimulation in Poor Responders Does Not Improve In Vitro Fertilization Outcome. Fertility and Sterility, 65, 961-965.

[17] Karande, V., Morris, R., Rinehart, J., Miller, C., Rao, R. and Gleicher, N. (1997) Limited Success using the "Flare" Protocol in Poor Responders in Cycles with Low Basal Follicle-Stimulating Hormone Levels during In Vitro Fertilization. Fertility and Sterility, 67, 900-903.

[18] Howles, C.M., Loumaye, E., Germond, M., Yates, R., Brinsden, P., Healy, D., et al. (1999) Does Growth Hormone-Releasing Factor Assist Follicular Development in Poor Responder Patients Undergoing Ovarian Stimulation for In Vitro Fertilization? Human Reproduction, 14, 1939-1943.

[19] $\mathrm{Pu}, \mathrm{D} ., \mathrm{Wu}, \mathrm{J}$. and Liu, J. (2011) Comparisons of GnRH Antagonist versus GnRH Agonist Protocol in Poor Ovarian Responders Undergoing IVF. Human Reproduction, 26, 2742-2749. https://doi.org/10.1093/humrep/der240

[20] Al-Inany, H. and Aboulghar, M. (2002) GnRH Antagonist in Assisted Reproduction: A Cochrane Review. Human Reproduction, 17, 874-885.

https://doi.org/10.1093/humrep/17.4.874 